\title{
El carácter metropolitano de la urbanización en México, 1900-1988
}

\section{Gustavo Garza*}

Es incuestionable la importancia de la ciudad para el crecimiento económico por ser el espacio donde se realizan las actividades secundarias y terciarias y, por ende, donde se acumula el capital. En México, sin embargo, no existen investigaciones significativas que analicen los nexos entre el desarrollo económico y la urbanización. En espera de que se realicen en el futuro, en este artículo se plantea únicamente sus vínculos más generales para centrarnos en el análisis de los aspectos más relevantes del crecimiento urbano de México durante el siglo $\mathrm{Xx}$.

El rasgo fundamental de las 274 ciudades mexicanas en 1988 es su carácter preeminente. Aunque la sola presencia de la ciudad de México le conferiría una dimensión metropolitana a la urbanización del país, este fenómeno se ve fortalecido por el surgimiento de otras 26 ciudades en diferentes etapas de metropolitanismo. No obstante que este proceso se pueda interpretar como evidencia de cierta dispersión territorial de la población, ocurre paralelamente un desarrollo urbano metropolitano dependiente del nuevo núcleo megalopolitano en torno a la ciudad de México.

A partir de 1982 el crecimiento económico se ha interrumpido, pero no así el proceso de urbanización. El escenario socioeconómico del sistema de ciudades mexicanas depende fundamentalmente de las posibilidades reales de vencer la crisis económica, retomando las anteriores tasas de crecimiento. De no hacerlo en un futuro previsible, y todo parece indicar que así será, la situación económica, social y urbanística de la población urbana será cada vez más insostenible.

La interacción entre el desarrollo económico y la urbanización es universal. Sin embargo, su carácter específico presenta marcadas diferencias, aunque es posible hablar de la existencia de un patrón de urbanización para los países desarrollados y de otro para los subdesarrollados. En los primeros la urbanización, como ha sido entendida, prácticamente ha concluido al tener una población urbana que representa casi $100 \%$ de la total. No obstante, experi-

* Profesor-investigador del Centro de Estudios Demográficos y de Desarrollo Urbano de El Colegio de México. Este artículo es la versión en castellano de la ponencia "The Metropolitan Character of Urbanization in Mexico, 1900-1988", presentada en el International Symposium on Latin America Urbanization, realizado en la Universidad de Tsukuba, lapón, del 23 al 27 de octubre de 1989. Agradecemos al Prof. Mutsuo Yamada, coordinador del simposium, su consentimiento para realizar esta publicación. 
mentan una importante transformación denominada "suburbanización" por constituir una nueva estructuración polinuclear del espacio urbano con muy reducidas densidades de población y extensas áreas verdes, como es el caso de las ciudades estadunidenses. Las naciones del tercer mundo y las socialistas se encuentran, por el contrario, experimentando aún acelerados procesos de urbanización dentro de sus muy diferentes niveles alcanzados. La urbanización del subdesarrollo se caracteriza, a su vez, por mantener o aun acentuar su característico patrón de alta primacía urbana y por las grandes carencias sociales e infraestructurales que existen en sus ciudades.

Es incuestionable la importancia de la ciudad para el desarrollo económico por ser el espacio donde se acumula el capital y se realizan las principales inversiones. En los países del tercer mundo de mayor desarrollo relativo, alrededor del $80 \%$ del crecimiento anual del producto nacional se genera en las ciudades (véase, Armstrong y McGee, 1985:219; Harris, 1988:103). Sorprende, por tanto, que la gran mayoría de sus gobiernos no han sido conscientes de la necesidad de establecer políticas urbanas acordes a los requerimientos del desarrollo económico.

La acelerada urbanización de México durante el siglo Xx ha sido resultado de la rápida industrialización ocurrida hasta 1982. La relación estructural entre ambos procesos no ha sido investigada, por lo que en este trabajo nos limitaremos a describir sus nexos principales y analizar las características más relevantes del crecimiento urbano del país en lo que va del presente siglo.

\section{Desarrollo económico, industrialización y urbanización}

El desarrollo económico de México hasta inicios de los años ochenta fue resultado de una política de sustitución de importaciones iniciada en la década de los treinta, que reemplazó al patrón agroexportador vigente desde la segunda mitad del siglo XIX. No obstante, este último siguió existiendo subordinado al modelo industrial hasta agotarse totalmente en los cincuenta. La articulación de ambos patrones de 1930 a 1950 fue de gran importancia para el éxito relativo de la política de sustitución de importaciones.

El crecimiento económico de 1930 a 1980 fue importante. El Producto Interno Bruto (PIB) total entre 1930 y 1940 aumentó en términos reales a $3.1 \%$ anual; a $5.9 \%$ entre 1940 y 1950 ; a $6.2 \%$ en 1950-1960; y continuó avanzando hasta alcanzar $7.0 \%$ anual entre 1960 y 1970. La década 1970-1980 auguraba un mayor dinamismo 
por el gran aumento en los precios del petróleo y la rápida expansión de la explotación de los yacimientos en el país, pero la crisis de mediados de la década lo impidió, a pesar de lo cual la economía creció al $6.6 \%$. Este significativo crecimiento fue impulsado por la industria de transformación, que tuvo tasas sistemáticamente más elevadas que las del PIB total.

Los determinantes del desarrollo industrial durante el periodo 1930-1980 han sido analizados por diversos especialistas: intensificación de la reforma agraria entre 1933 y 1939 con la repartición de 23.5 millones de hectáreas; movilización de la fuerza de trabajo rural a las ciudades, principalmente a la de México; participación creciente del Estado en materia de infraestructura de comu nicaciones y urbana, sin la cual la rentabilidad del capital industrial no es posible; aumento considerable del crédito externo, que pasó de $6.7 \%$ de la inversión bruta fija estatal entre $1942-1946$ a $30 \%$ entre 1959-1962; fomento y captación del ahorro interno por medio de un conjunto de instituciones financieras oficiales (entre las que destaca Nacional Financiera, S.A. creada desde 1934 con objeto de financiar la inversión privada); estricta protección arancelaria para estimular el desarrollo de la industria del país; expansión de la capacidad de importar por el aumento del turismo internacional; absorción productiva de la creciente población urbana y rural que ensanchó el mercado interno nacional elevando la demanda agregada; mayor inversión extranjera directa en la industria; aumento de la demanda externa durante la segunda guerra mundial y la guerra de Corea; establecimiento de una política fiscal favorable a los sectores industriales; inversión creciente del Estado en empresas manufactureras; establecimiento de un conjunto de políticas de estímulos a la industrialización a través de exenciones fiscales y tasas de interés preferenciales; creación de un frente de organizaciones obreras, campesinas y populares bajo el control estatal (véase, Carmona de la Peña, 1979; Ramírez Brun, 1980; Vernon, 1977; Solís, 1970; Tello, 1979).

La distribución territorial del desarrollo y el acelerado crecimiento de la población permitió un significativo aumento en el número de ciudades. La jerarquía urbana resultante se caracterizó por la creciente importancia de la ciudad de México.

La capital del país ha constituido el núcleo principal de la economía nacional y su dinámica económica es el principal determinante de su desarrollo demográfico y urbanístico que posteriormente se analiza. La ciudad debe considerarse, entonces, como una unidad económica que produce, distribuye y consume mercancías.

En 1940 la ciudad de México absorbía 7.9\% de la población 
nacional, pero su importancia económica era considerablemente mayor. En ese año el PIB estimado para el área metropolitana de la ciudad de México (AMCM) fue de 107 miles de millones de pesos que significaron $33.5 \%$ del total nacional. En términos sectoriales la urbe participa con $36.2 \%$ del sector secundario y $46.2 \%$ del sector servicios (véase el cuadro 1).

Durante 1950-1970 la contribución del AMCM al PIB nacional aumentó de $31.2 \%$ a $34.6 \%$. El incremento más importante ocurrió entre 1950-1960 debido básicamente al dinamismo del sector industrial el cual, según las estimaciones del cuadro 1, alcanzó $37.5 \%$ del total nacional correspondiente. Aunque el porcentaje del AMCM en la población nacional también aumentó durante el período -alcanzando $18.2 \%$ en 1970 -, su participación en la economía nacional se mantuvo muy por arriba de la importancia demográfica.

De 1970 a 1980 la tasa de crecimiento del PIB en México fue todavía muy importante (6.6\% en términos reales), prosiguiendo la significativa dinámica económica que se había experimentado desde los años treinta, cuando inició la estrategia de sustitución de importaciones. En esta década la participación de la ciudad de México en el PIB nacional continuó aumentando hasta alcanzar $37.8 \%$ en 1980 . En forma paralela, su crecimiento demográfico fue mayor que el nacional, y en 1980 absorbe $20.8 \%$ y $37.0 \%$ de la población total y urbana del país, respectivamente.

Finalmente, entre 1980-1985 el desarrollo económico nacional y de la ciudad de México fue insignificante, pero fue menor en esta última que reduce su participación al $35.3 \%$ (véase el cuadro 1). No obstante su contracción económica, suele suponerse que el crecimiento demográfico de la capital del país es aún elevado -alrededor de 3\% anual- y que su población total fue de cerca de 19 millones en 1988.

Considerando la importancia de la ciudad de México para la economía nacional, sorprende constatar que no existe ningún análisis comprehensivo de su estructura económica. Las cifras anteriores de sus variables macroeconómicas son estimaciones muy gruesas según su población económicamente activa y los datos del PIB por entidades federativas, por lo que deben utilizarse con las precauciones pertinentes. La industria manufacturera, sin embargo, está más sistemáticamente investigada con base en la información de los censos económicos (véase, Garza, 1985; Garza y Aguilar, 1988).

En 1930 México tenía 46.8 miles de establecimientos industriales de los cuales menos del $7 \%$ se localizaban en la ciudad de México. Para 1950 su número aumentó a 63.5 miles en el país, de 
los cuales 12.7 se ubicaron en la capital nacional, representando $20.0 \%$. Durante los siguientes 20 años, la producción industrial mantuvo su dinámico crecimiento alcanzando tasas de $7.3 \%$ anual entre 1950 a 1960 y $8.9 \%$ en 1960 a 1970 , mientras la concentración espacial en la ciudad de México se elevaba al $27.9 \%$ según número de establecimientos (33.2 miles en un total nacional de 119 mil, véase el cuadro 2). La mayor concentración de firmas industriales ocurre en 1980, cuando la ciudad de México tuvo 38.5 miles que representan $29.5 \%$ del total nacional. Es importante destacar el crecimiento absoluto de 8561 empresas entre 1960 a 1970 y de 5307 entre 1970 a 1980 , por constituir nuevos establecimientos que fomentan el crecimiento urbano por su demanda directa e indirecta de fuerza de trabajo, servicios y actividades comerciales.

Aún más impresionante es la concentración industrial en la ciudad de México en términos del producto. De 1930 a 1980 fue elevando ininterrumpidamente su participación de la industria nacional: $28.5 \%$ en $1930 ; 32.1 \%$ en $1940 ; 40.0 \%$ en $1950 ; 46.0 \%$ en $1960 ; 46.8 \%$ en 1970 y $48.0 \%$ en 1980 (véase el cuadro 2). En este último año, por ende, una de las 229 ciudades que conformaban la jerarquía urbana nacional producía casi la mitad del total de mercanías industriales elaboradas en la república.

Durante los años ochenta México ha experimentado su más severa crisis económica de la historia moderna. Entre 1983 a 1988 el PIB se redujo en $-0.2 \%$ anual, al mismo tiempo que la industria nacional experimentaba una disminución de $-0.4 \%$. ¿En el contexto de esta crisis la industria de la ciudad de México fue menos afectada que su contraparte nacional? Dada su más diversificada estructura industrial, podría pensarse que la ciudad es menos vulnerable a las fluctuaciones económicas porque mientras algunas ramas industriales reduzcan su producción, otras la aumentarán y, en promedio, su situación económica tenderá a ser mejor que el de otras ciudades del país. Las cifras industriales disponibles para 1985 señalan, sin embargo, que ocurre lo contrario.

Efectivamente, en el quinquenio 1980 a 1985 la ciudad de México experimentó, por primera vez desde 1930, una reducción absoluta de sus empresas industriales de 38.4 a 33.1 miles, que fue más acentuada que la del país en su conjunto (véase el cuadro 2). No fue posible obtener información de la producción industrial, pero el personal ocupado decrece de 1.1 millones en 1980 a 844 mil en 1985, describiendo dramáticamente la crisis económica de la urbe. Esta situación es uno de los determinantes fundamentales del agravamiento de sus problemas urbanos, tales como el desempleo, el déficit habitacional, el aumento de zonas marginadas, la 


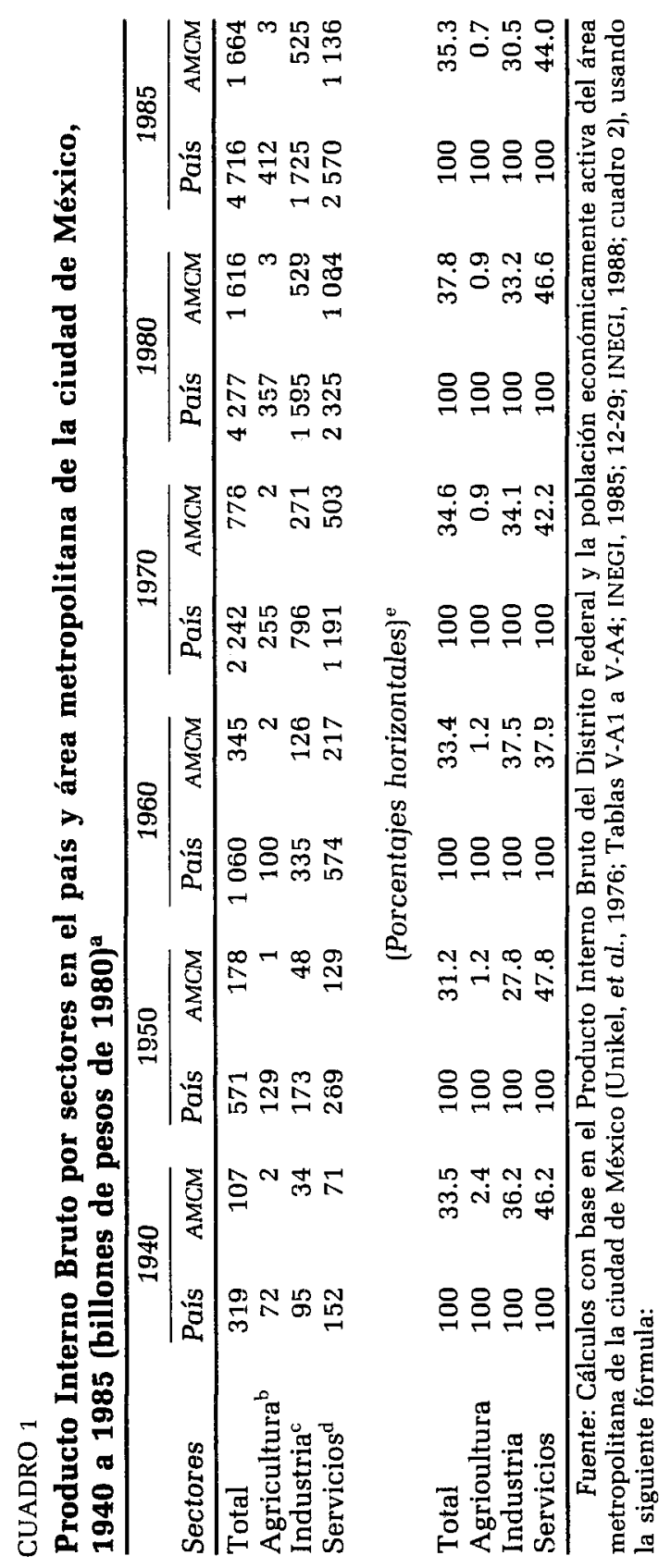




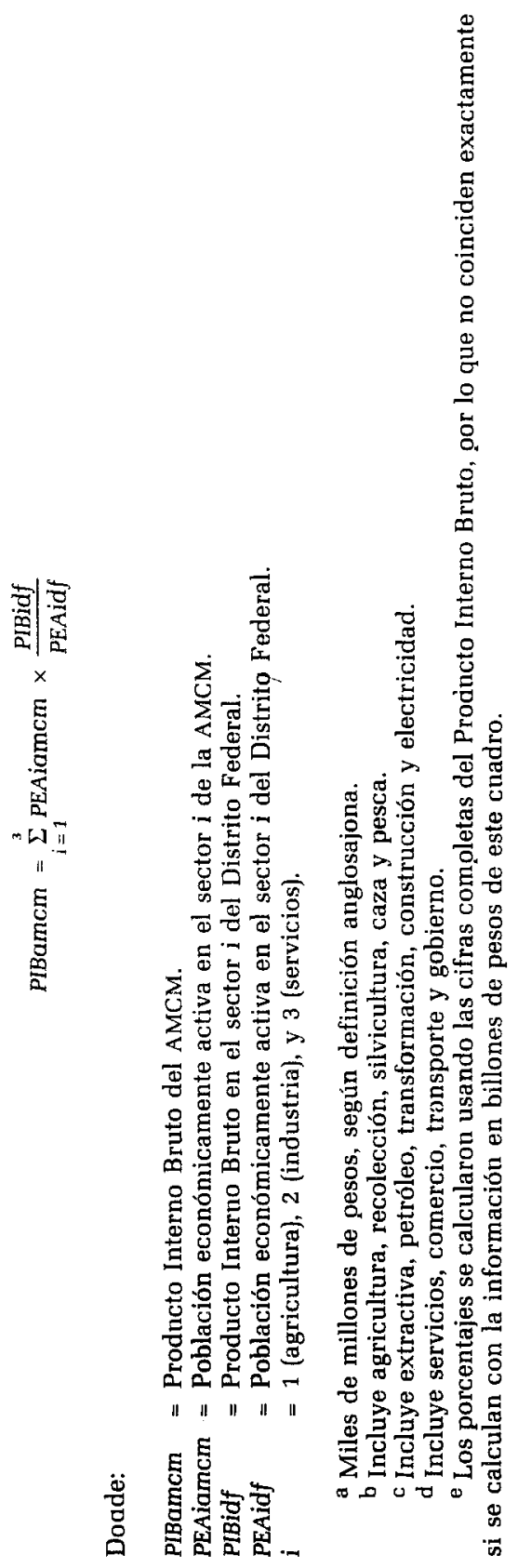


CUADRO 2

Establecimientos industriales, personal ocupado y producción bruta en el país y en la ciudad de México, 1930 a 1985

\begin{tabular}{|c|c|c|c|c|c|c|}
\hline \multirow[b]{2}{*}{ Año } & \multicolumn{2}{|c|}{$\begin{array}{c}\text { Establecí- } \\
\text { mientos }\end{array}$} & \multicolumn{2}{|c|}{ Personal } & \multicolumn{2}{|c|}{$\begin{array}{c}\text { Producción } \\
\text { bruta }^{\mathbf{a}}\end{array}$} \\
\hline & Total & $\%$ & Total & $0 \%$ & Total & $\%$ \\
\hline 1930 & & & & & & \\
\hline País & 46830 & 100.0 & 284794 & 100.0 & 2960 & 100.0 \\
\hline $\begin{array}{c}\text { Ciudad de México } \\
1940\end{array}$ & 3180 & 6.7 & 54105 & 19.0 & 843 & 28.5 \\
\hline País & 56314 & 100.0 & 362536 & 100.0 & 6800 & 100.0 \\
\hline $\begin{array}{c}\text { Ciudad de México } \\
1950\end{array}$ & 4920 & 8.7 & 89358 & 24.6 & 2182 & 32.1 \\
\hline País & 63544 & 100.0 & 626285 & 100.0 & 18289 & 100.0 \\
\hline $\begin{array}{c}\text { Ciudad de México } \\
1960\end{array}$ & 12704 & 20.0 & 156697 & 25.0 & 7324 & 40.0 \\
\hline País & 82352 & 100.0 & 884927 & 100.0 & 29452 & 100.0 \\
\hline $\begin{array}{c}\text { Ciudad de México } \\
1970\end{array}$ & 24624 & 29.9 & 406905 & 45.9 & 138535 & 46.0 \\
\hline País & 118993 & 100.0 & 1596816 & 100.0 & 69323 & 100.0 \\
\hline $\begin{array}{c}\text { Ciudad de México } \\
1980\end{array}$ & 33185 & 27.9 & 658275 & 41.2 & 32437 & 46.8 \\
\hline País & 130494 & 100.0 & 2258990 & 100.0 & 122740 & 100.0 \\
\hline $\begin{array}{c}\text { Ciudad de México } \\
1985\end{array}$ & 38492 & 29.5 & 1059182 & 46.9 & 58943 & 48.0 \\
\hline País & 127539 & 100.0 & 2303600 & 100.0 & n.d. & n.d. \\
\hline Ciudad de México & 33143 & 26.0 & 843800 & 36.6 & n.d. & n.d. \\
\hline
\end{tabular}

Fuente: I, III, V. VII, IX, XI, y XII, Censos industriales, Dirección General de Estadística, México.

${ }^{\mathrm{a}}$ (millones de pesos, $1980=100$ )

n.d.: No disponible.

contaminación atmosférica, así como la inadecuada dotación de servicios de agua, drenaje, transporte, vigilancia, recolección de basura, etcétera.

Antes de la crisis, la expansión económica de la ciudad de México estimuló el crecimiento acelerado de las ciudades a su alrededor que conforman un subsistema urbano altamente integrado. Este proceso reducía la tendencia hacia mayores niveles de concentración de población e industrial en el núcleo urbano central constituido por la capital, originando el surgimiento de un conglomerado megalopolitano que será posteriormente analizado. 
Esta grave contracción en la actividad económica ha tenido un impacto en el nivel de vida de la población mexicana que ha visto reducir su poder adquisitivo en $53.2 \%$ entre 1982 a 1988 . A nivel urbano no existe ningún estudio riguroso sobre su impacto en la dinámica de crecimiento de las ciudades. Sin embargo, en lo que sigue se estima el desarrollo urbano hasta 1988 pa ra dar una imagen más actualizada del fenómeno, la cual podrá ser contrastada con la información censal de 1990.

\section{Etapas, niveles y tasas de urbanización}

La urbanización es un proceso de transformación secular de las estructuras rurales en urbanas. Según esta concepción, la u rbanización se caracteriza por el desarrollo incesante de la división social del trabajo, que transfiere la fuerza laboral agrícola hacia actividades secundarias y terciarias; la modernización tecnológica de los procesos de producción; la creciente diferenciación política y social entre los sectores rural y urbano; y por dar origen a la ciudad estimulando la multiplicación de su número y el aumento del tamaño de las urbes existentes.

En el caso mexicano, el desarrollo de la división social del trabajo se refleja nítidamente en la transformación de la estructura de la producción durante el siglo Xx: el sector secundario aumenta sistemáticamente su importancia en el producto interno bruto de $16.7 \%$ en 1900 a $34.9 \%$ en 1985 ; como corolario, las actividades agropecuarias reducen su absorción del producto de $25.8 \%$ en 1900 a $9.2 \%$ en 1985 .

Estos cuatro porcentajes reflejan la profunda transformación de la estructura de la producción resultante de un proceso de desarrollo económico que ocurre simultáneamente con la urbanización de la sociedad mexicana. Dado que las crecientes actividades secundarias y terciarias no requieren directamente de la tierra como insumo ni de la dispersión de la población como ocurre con las actividades primarias, paulatinamente se concentran en ciudades, dando lugar a su multiplicación y acelerado crecimiento. El éxodo rural-urbano de la fuerza de trabajo que origina este proceso determina en buena medida al desarrollo urbano, pues sin migración difícilmente ocurre la urbanización.

El proceso de urbanización resultante ha sido ininterrumpido en lo que va del siglo, aunque se ha manifestado a diferentes ritmos. En 1900 la población total de México era de 13.6 millones, de los cuales 1.4 vivia en ciudades y 12.2 en localidades rurales, esto es, únicamente $\mathbf{1 0 . 5 \%}$ de la población era urbana (por ejem- 
plo, población viviendo en localidades de 15 mil y más habitantes). La Revolución mexicana interrumpe el crecimiento de la población total que se reduce de 15.2 millones en 1910 a 14.3 en 1921, pero el proceso de urbanización continua y la población urbana aumenta de 1.8 a 2.1 millones de habitantes, elevando el nivel de urbanización de $11.7 \%$ a $14.7 \%$, respectivamente (véase el cuadro 3).

A partir de 1940 se consolidan en el poder los grupos políticos surgidos del movimiento armado y la población entra en un periodo de acelerado crecimiento. El desarrollo económico que se inicia en esa época se manifiesta demográficamente en una drástica disminución de la mortalidad, mientras que la natalidad se mantiene elevada. Esto produce que la población total que aumentó a $1.7 \%$ anual entre 1930 a 1940 eleve su crecimiento anual a $3.4 \%$ en 1960 a 1970 y a $3.3 \%$ entre 1970 a 1980 , reduciéndolo al parecer de 2.0 a $2.5 \%$ después de 1980 . De esta forma, para 1988 se estima que México tiene 82.7 millones de habitantes siendo, después de Brasil, el país más poblado de América Latina.

La población urbana aumentó a tasas mucho más elevadas que las de la población total. En 1940 se tienen 3.9 millones de mexicanos viviendo en ciudades, cantidad que casi se cuadruplica para 1960 cuando 14.4 millones habitan en ciudades y se inicia en México un proceso de urbanización de carácter metropolitano (véase el cuadro 3). Esto ocurre entre 1950 a 1960 cuando la ciudad de México se transforma en zona metropolitana al extender su mancha urbana del Distrito Federal al Estado de México, la principal entidad federativa que lo rodea. Cabría aclarar que a partir de 1960 la población urbana de los cuadros 3 y 4 incluyen los habitantes de las áreas metropolitanas que van surgiendo a medida que se extiende el carácter metropolitano de la urbanización en México.

Lo anterior se refleja en un aumento muy significativo del grado de urbanización que más que se duplica al pasar de $20.0 \%$ en 1940 a $41.2 \%$ en 1960 . A partir de este último año se reduce el ritmo de urbanización y su nivel pasa de $49.4 \%$ en 1970 a $56.2 \%$ en 1980. Se estima que sea de $63.8 \%$ en 1988 (véase el cuadro 3).

Hacia finales de la década de los ochenta, por ende, México adquiere un carácter más definidamente urbano que ha trasladado la problemática que enfrenta el Estado mexicano del campo a la ciudad. Los problemas de la tenencia de la tierra en las ciudades, el transporte, la gran insuficiencia de servicios e infraestructura urbana, la marginalidad y el desempleo, la contaminación de los ecosistemas urbanos, la inseguridad pública, entre otros dramas, han sustituido en gravedad a los ancestrales problemas rura- 


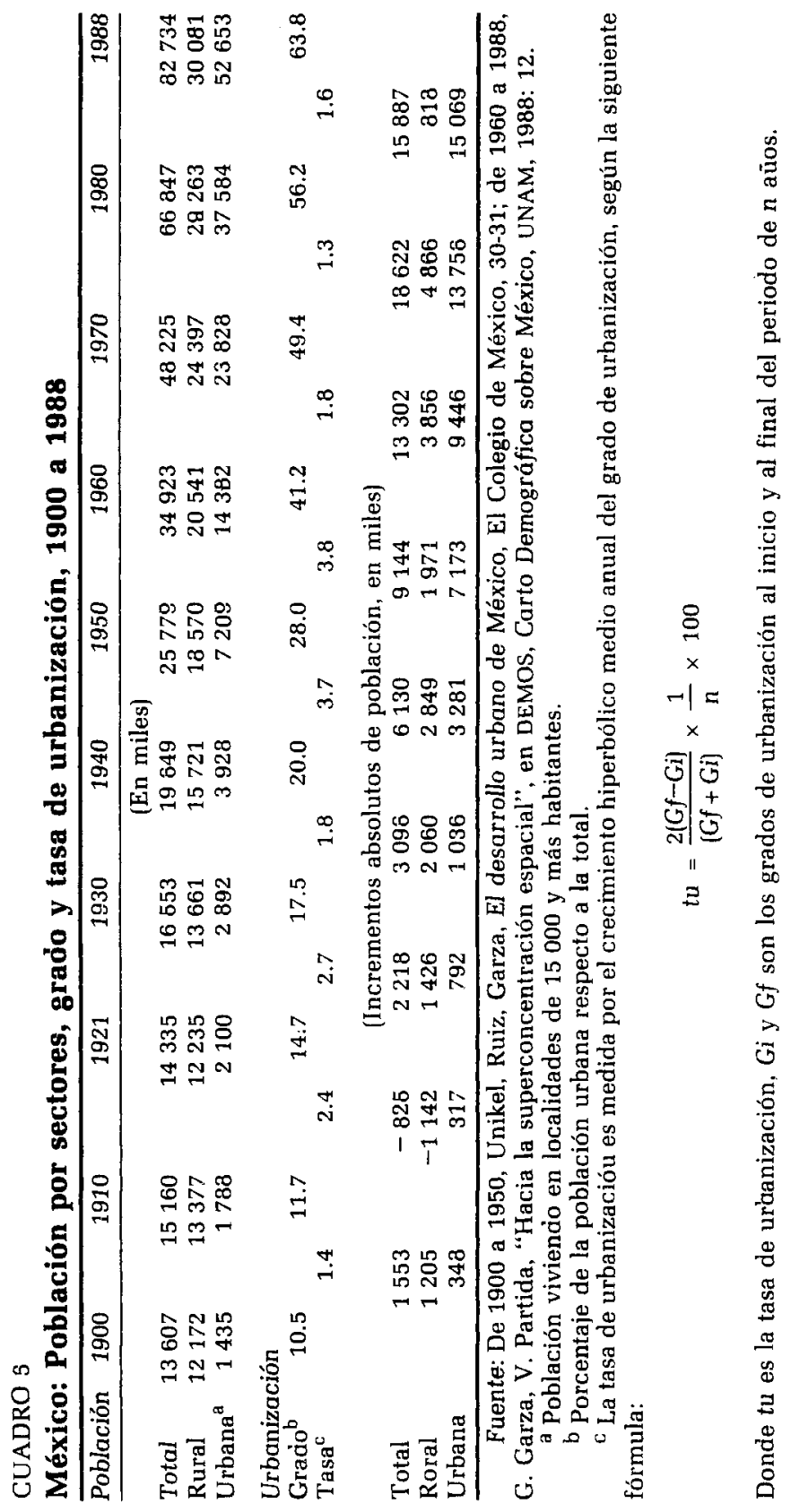


les que, no obstante, siguen acentuándose dramáticamente.

Según el ritmo del desarrollo urbano en México, los 88 años transcurridos del siglo XX se podrían dividir en dos grandes etapas: a) de urbanización lenta de 1900 a 1940; b) urbanización acelerada de 1940 a 1988. La tasa de urbanización anual en los 40 años del primer periodo fue de $1.6 \%$, elevándose a $2.2 \%$ en los 48 años transcurridos entre 1940 a 1988 . Sin embargo, el ritmo de urbanización dista mucho de ser uniforme en ambas etapas, observándose su aumento entre 1910-1930 del primer periodo, y una marcada disminución después de 1960 en el segundo (véase el cuadro 3).

Lo anterior se debe en buena medida al significativo cambio observado en las magnitudes absolutas de la población urbana que a partir de 1980 supera a su contraparte rural, representando un total de 37.6 millones de habitantes (véase el cuadro 3). De esta forma, a pesar de la reducción de las tasas de urbanización después de 1960 , ocurre un creciente aumento absoluto de la población urbana que supera ligeramente al aumento de la rural a partir de 1940, pero que hacia 1988 lo hace en forma abrumadora. Así, entre 1970 a 1980 la población rural crece en 4.9 millones de personas, mientras que la urbana lo hace en 13.8. Para 1980 a 1988 se estiman estas magnitudes en 0.8 y 15.1 millones de personas, respectivamente (véase el cuadro 3). En verdad, México se encuentra imposibilitado para enfrentar en forma razonable los requerimientos de empleo, infraestructura y servicios urbanos de estos incrementos de población.

\section{El sistema de ciudades}

En 1900 el sistema de ciudades en México estaba constituido por 33 localidades en las cuales vivían 1.4 millones de personas. Su evolución en los 88 años transcurridos se puede analizar siguiendo las dos etapas principales del proceso de urbanización del país.

Durante la etapa de urbanización lenta, esto es, de 1900 a 1940, surgieron 22 nuevas ciudades a razón de 0.5 anuales, aumentando la población urbana a 3.9 millones en 1940 (véase el cuadro 4). El aumento de 2.5 millones de población urbana surge de tres diferentes fuentes: a) por la reclasificación de localidades rurales a urbanas al sobrepasar los $\mathbf{1 5}$ mil habitantes (ciudades reclasificadas); b) por la incorporación de población de localidades rurales durante la expansión del área de las ciudades; y, c) por el crecimiento natural y por migración de la población de las ciudades existentes. 
CUADRO 4

México: distribución de la población urbana por tamaño de ciudades, 1900 a 1988

\begin{tabular}{|c|c|c|c|c|c|c|c|}
\hline Años & $\begin{array}{c}\text { Urbano } \\
\text { total }\end{array}$ & $\begin{array}{c}15000 \\
a \\
19999\end{array}$ & $\begin{array}{c}20000 \\
a \\
49999\end{array}$ & $\begin{array}{c}50000 \\
a \\
99999\end{array}$ & $\begin{array}{c}100000 \\
a \\
499999\end{array}$ & $\begin{array}{c}500000 \\
a \\
999999\end{array}$ & $\begin{array}{c}1000000 \\
\text { y más }\end{array}$ \\
\hline \multicolumn{8}{|l|}{1900} \\
\hline Población ${ }^{\mathbf{a}}$ & 1435 & 173 & 536 & 280 & 446 & - & - \\
\hline Ciudades ${ }^{b}$ & 33 & 10 & 17 & 4 & 2 & - & - \\
\hline \multicolumn{8}{|l|}{1910} \\
\hline Población & 1783 & 115 & 715 & 363 & 590 & - & - \\
\hline Ciudades & 36 & 7 & 22 & 5 & 2 & - & - \\
\hline \multicolumn{8}{|l|}{1921} \\
\hline Población & 2100 & 201 & 560 & 534 & 143 & 662 & - \\
\hline Ciudades & 39 & 12 & 17 & 8 & 1 & 1 & - \\
\hline \multicolumn{8}{|l|}{1930} \\
\hline Población & 2892 & 275 & 564 & 575 & 429 & - & 1049 \\
\hline Ciudades & 45 & 16 & 17 & 8 & 3 & - & \\
\hline \multicolumn{8}{|l|}{1940} \\
\hline Población & 3928 & 304 & 694 & 589 & 781 & - & 1560 \\
\hline Ciudades & 55 & 18 & 23 & 8 & 5 & - & \\
\hline \multicolumn{8}{|l|}{1950} \\
\hline Población & 7209 & 392 & 1210 & 808 & 1927 & - & 2872 \\
\hline Ciudades & 84 & 22 & 39 & 12 & 10 & - & \\
\hline \multicolumn{8}{|l|}{1960} \\
\hline Población & 14382 & 559 & 1271 & 1956 & 3591 & 1596 & 5409 \\
\hline Ciudades & 119 & 32 & 41 & 26 & 17 & 2 & \\
\hline \multicolumn{8}{|l|}{1970} \\
\hline Población & 23828 & 707 & 1950 & 1510 & 7284 & 732 & 11645 \\
\hline Ciudades & 166 & 41 & 65 & 21 & 35 & 1 & \\
\hline \multicolumn{8}{|l|}{1980} \\
\hline Población & 37584 & 1010 & 2876 & 1633 & 10230 & 2553 & 19282 \\
\hline Ciudades & 229 & 59 & 94 & 24 & 44 & 4 & - \\
\hline \multicolumn{8}{|l|}{1988} \\
\hline Población & 52653 & 1137 & 3789 & 2287 & 11092 & 5658 & 28690 \\
\hline Ciudades & 274 & 61 & 122 & 31 & 46 & 8 & \\
\hline
\end{tabular}

Fuente: 1900 a 1950 de Unikel, Ruiz, Garza, El desarrollo urbano de México, El Colegio de México, 1976:30-31; 1960 a 1988 de G. Garza y V. Partida, "Hacia la superconcentración espacial", en DEMOS, Carta demográfica sobre México, UNAM, 1988:12.

En miles de habitantes.

${ }^{\mathrm{b}}$ Localidades con 15 mil y más habitantes.

Al inicio del proceso de urbanización en México, las dos primeras formas de crecimiento fueron importantes, pero en la etapa de crecimiento urbano acelerado dejaron de serlo. Así, por ejem- 
plo, los cinco centros que fueron reclasificados entre 1900 a 1910 contribuyeron con $38.2 \%$ del crecimiento en la población urbana de esa década. La importancia de la reclasificación de localidades se redujo drásticamente entre 1940 a 1950, cuando los 30 centros incorporados contribuyeron con $19.2 \%$ y fue despreciable entre 1960 a 1970 cuando las 55 localidades reclasificadas únicamente participaron con 4.2\% (Unikel, 1977:494). En la segunda etapa de la urbanización, entonces, las localidades reclasificadas tuvieron muy poco impacto en el crecimiento total de la población urbana.

En el casi medio siglo de urbanización acelerada que va de 1940 a 1988, emergen 219 nuevas ciudades para conformar un sistema de 274 urbes en 1988. En este lapso la población urbana aumentó en 48.7 millones de habitantes, para hacer un total de 52.6 millones (véase el cuadro 4). De esta suerte, este periodo de urbanización acelerada se caracteriza por tener un promedio anual de 4.6 nuevas ciudades y un poco más de 1 millón de nuevos habitantes. Este crecimiento se explica en $80 \%$ por la dinámica de las ciudades existentes y en $20 \%$ por las reclasificadas y las localidades incorporadas por la expansión de las manchas urbanas.

El sistema urbano se suele jerarquizar según la distribución de la población por tamaños de ciudades. Se le denomina preeminente cuando la ciudad principal supera por varias veces a la que le sigue (convencionalmente más de tres veces); y de rangotamaño cuando la primera ciudad duplica a la segunda, triplica a la tercera, cuadruplica a la cuarta y, en general, es n veces mayor que la ciudad de rango $\mathrm{n}$. Se podría hablar de una jerarquía urbana intermedia cuando la distribución de la población se encuentre entre ambas posibilidades.

A finales del siglo XVIII y a principios del XIX, la ciudad de México no acusaba un fuerte predominio sobre el resto de ciudades del México colonial, a pesar de ser la más importante de la Nueva España. De esta forma el índice de primacía para 1790 era únicamente de 1.3, reflejando que la ciudad de México tenía únicamente $30 \%$ más habitantes que Puebla, que constituía la segunda ciudad de la época. Al principio del siglo XIX el índice se eleva a 2.0, ajustándose perfectamente a un sistema de ciudades de tipo rango-tamaño (véase el cuadro 5).

En México, el capitalismo industrial como modo de producción dominante se consolidó hacia las últimas dos décadas del siglo XIX y es a partir de esta época cuando la ciudad de México inicia su acelerado crecimiento. De esta suerte, para 1900 el índice de primacía se eleva a 4.4 y sigue aumentando hasta alcanzar 7.2 en 1950, para estabilizarse en alrededor de seis en los decenios siguientes (véase el cuadro 5). 
CUADRO 5

México: índice de primacía de dos ciudades, 1790 a 1985

\begin{tabular}{cc}
\hline Año & Índice $^{\mathrm{a}}$ \\
\hline 1790 & 1.3 \\
1803 & 2.0 \\
1900 & 4.4 \\
1910 & 3.9 \\
1921 & 4.3 \\
1930 & 5.7 \\
1940 & 6.5 \\
1950 & 7.2 \\
1960 & 6.1 \\
1970 & 6.1 \\
1980 & 6.1 \\
1985 & 6.2 \\
\hline
\end{tabular}

Fuente: 1790 y 1803 en G. Garza, El proceso de industrialización en la ciudad de México, El Colegio de México, 1985: 69; 1900 a 1970 en Unikel, Ruiz, Garza, El desarrollo urbano de México, El Colegio de México 1976:57; 1980 en C. Ruiz y A.M. Tepichín, "Preeminencia de la ciudad de México 1940-1980", en G. Garza (comp.), Atlas de la ciudad de México, México, D.F., 1987; y 1985 de, United Nations, The prospects of world urbanization, New York, 1987: Tabla 6.

a El índice se calcula de la siguiente manera:

$$
I p=\frac{P 1}{P 2}
$$

donde P1 es la población de la ciudad de México y P2 es la población de Puebla en 1790 y 1803, y de Guadalajara desde 1900.

Hacia finales del siglo xx, por tanto, se mantiene en México un sistema urbano altamente preeminente que implica una elevada concentración territorial de la población y las actividades económicas. Esto representa, a nuestro parecer, un freno al desarrollo económico pues al concentrar el grueso de la inversión pública impide que se utilicen los recursos y fuerza de trabajo de vastas regiones del país.

La urbanización acelerada experimentada en México ocurrió en un primer momento concentrada en la ciudad capital. Sin embargo, la población se empieza a dispersar relativamente hacia otras metrópolis emergentes, entre las cuales sobresalen Guadalajara y Monterrey.

En 1900 el $49.4 \%$ de la población urbana vivía en ciudades menores a los 50 mil habitantes, este porcentaje se reduce a $25.4 \%$ en 1940 y a únicamente $9.4 \%$ en 1988 . En contrapartida, si en el primer año no existía ninguna localidad mayor de $\mathbf{5 0 0}$ mil habitantes, para el segundo $39.7 \%$ de la población urbana vive en ese 
estrato de población, porcentaje que se eleva a $65.2 \%$ en la actualidad (véase el cuadro 4).

La urbanización de corte metropolitano se extiende hacia otros núcleos de población formando nuevas áreas metropolitanas que en 1980 totalizan 26. Así, a la ciudad de México, Guadalajara y Monterrey se agregan nuevas áreas metropolitanas como las de Puebla, Torreón, León, Orizaba, Tampico, Toluca, entre otras, imprimiéndolé indefectiblemente un carácter metropolitano a la urbanización de México.

Algunos especialistas afirman que la emergencia de nuevas áreas metropolitanas es síntoma del agotamiento del proceso de concentración urbana en una sola metrópoli y que se inicia un proceso de descentralización espontáneo siguiendo la tendencia observada en algunos países desarrollados, principalmente en los Estados Unidos de América. Sin embargo, esto no significa necesariamente la culminación del proceso concentrador, pudiendo implicar sólo un cambio en el ámbito de concentración al pasar de un nivel metropolitano a otro megalopolitano, como se analiza en el siguiente apartado.

\section{La megalópolis de la ciudad de México}

La cristalización de una metrópoli es resultado de siglos de evolución. Aparentemente por razones religiosas, el conquistador español Hernán Cortés decidió construir el centro del imperio español sobre las ruinas de Tenochtitlán, la ciudad que destruyó durante la guerra de conquista. En los inicios de 1522 se inició la construcción de una ciudad que posteriormente (1548) fue bautizada como "La Muy Noble, Insigne y Muy Leal e Imperial Ciudad de México". En sus inicios como ciudad española estuvo constituida por 30 mil personas, tanto nativos como españoles.

Hacia finales del periodo colonial, en 1803, la ciudad de México contaba con 137 mil habitantes, los cuales aumentaron a 180 mil en 1810, en los inicios de la guerra de Independencia. Desde entonces y hasta la consumación de ésta en 1821, muchos españoles emigraron de la capital y su población se redujo a 165 mil en 1823.

México logra su independencia en 1821 y durante las primeras décadas como nación, la ciudad de México creció lentamente alcanzando a tener 200 mil personas en 1855 y 230 mil en 1877, cuando la tasa de crecimiento de su población empieza a ser más alta que la nacional. En los inicios del siglo XX, en 1900, la capital posee 345 mil habitantes e inicia un acelerado crecimiento urbano 
que la conduce, al final de los años ochenta, a ser probablemente la urbe más poblada del planeta. Durante este proceso la ciudad de México ha pasado por cuatro etapas de desarrollo que se sintetizan a continuación.

Primera etapa: crecimiento del núcleo central (1900 a 1930)

En una primera etapa de metropolitanismo, el área comercial aumenta su población residente en términos absolutos y relativos, así como la fuerza de trabajo que diariamente se dirige al "centro". Para el caso concreto de la ciudad de México, esta etapa cubre el periodo de 1900 a 1930 , cuando siendo una urbe relativamente pequeña de 345 mil habitantes en 1900, pasa a 1 millón 29 mil habitantes en 1930. El área urbana propiamente dicha registró una tasa de crecimiento de $3.3 \%$ anual, mientras que el Distrito Federal lo hacía a $2.6 \%$.

Sin embargo, en 1930 se inicia un proceso de expansión fuera de las 12 divisiones (cuarteles) en donde se localizaba toda la ciudad. Hasta 1921 éstos tenían 100\% de su población, pero en 1930 principia la expansión hacia las delegaciones de Coyoacán y Azcapozalco que absorben $2 \%$ de la población y en la parte central reside el $\mathbf{9 8 \%}$ restante. Ocurre, así, el crecimiento hacia las delegaciones que rodean a la ciudad central (véase el cuadro 6).

\section{Segunda etapa: expansión periférica (1930 a 1950)}

En los años treinta se inicia un segundo periodo de expansión territorial de la ciudad de México. Este se caracteriza por el crecimiento más acelerado de las delegaciones del Distrito Federal que rodean a los 12 cuarteles que definían a la ciudad central antes de 1930. Así, mientras la ciudad central crece de 1930 a 1940 al 3.4\% anual, 6 delegaciones del Distrito Federal y un municipio del Estado de México, que conforman un primer anillo de unidades administrativas que la rodean, lo hacen al 5.4\% anual. De 1940 a 1950 esta diferencia se acentúa significativamente y ambas tasas son de $4.3 \%$ y $10.3 \%$, respectivamente. Como corolario, la ciudad central pierde aceleradamente importancia respecto al área urbana de la ciudad de México, reduciendo su participación de $98 \%$ en 1930 a $78.3 \%$ en 1950 (véase el cuadro 6).

Esta segunda etapa marca el inicio de la descentralización del comercio, servicios y población del centro hacia las unidades administrativas periféricas. No obstante que esta expansión ocurre 


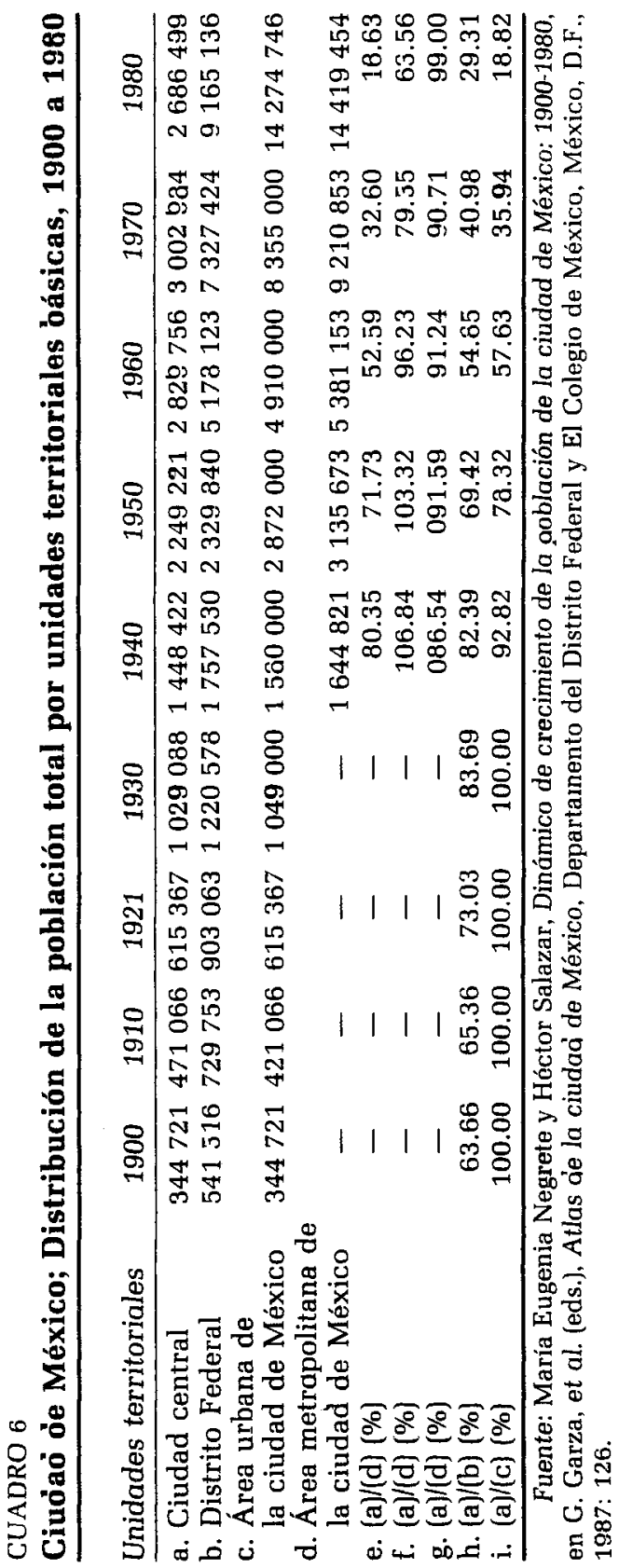


casi exclusivamente dentro del territorio del Distrito Federal, alcanza los límites de su superficie en el norte, por lo que 1950 representa la culminación de este periodo del crecimiento de la ciudad de México.

Tercera etapa: dinámica metropolitana (1950 a 1980)

En este lapso el AUCM rebasó los límites del Distrito Federal por el norte, extendiéndose hacia los municipios de Tlanepantla, Naucalpan y Ecatepec, dando lugar al surgimiento del área metropolitana de la ciudad de México (AMCM). Desde entonces se diferencian los conceptos de AUCM y AMCM. Para 1950 sólo Tlanepantla constituye parte del AMCM, pero en 1960 se le incorporan Naucalpan, Chimalhuacán y Ecatepec. Estos municipios del Estado de México registran una importante expansión demográfica al localizarse en su territorio gran parte de las nuevas empresas industriales que siguen un patrón de desconcentración industrial del centro de la ciudad hacia su periferia norte. De esta forma, entre 1950 a 1960 aumentaron en $10.3 \%$ anual su población, mientras que el distrito central lo hacía a $2.4 \%$. Éste continúa perdiendo rápidamente importancia llegando a absorber sólo $57.6 \%$ de la población del AUCM (véase el cuadro 6).

De 1960 a 1970 se agrega al AUCM los municipios de Nezahualcóyotl (creado en 1964 con parte del territorio de los municipios de Chimalhuacán, Texcoco y Ecatepec), la Paz, Zaragoza, Tultitlán, Coacalco, Cuautitlán y Huixquilucan. Éstos experimentaron $14.3 \%$ de crecimiento anual, consolidando el proceso metropolitano en la parte del estado de México que empieza a adquirir una creciente importancia conteniendo $20.4 \%$ de la población total del AMCM en 1970.

El crecimiento urbano metropolitano hacia el estado de México continúa, agregándose en 1980 a la zona metropolitana ocho municipios adicionales de esa entidad: Chalco, Chiautla, Chicoloapan, Chiconcuac, Ixtapaluca, Nicolás Romero, Tecamac y Texcoco. De esta forma, para 1980 el AMCM queda constituida por las 16 delegaciones del Distrito Federal y 21 municipios del estado de México. De estos últimos, 17 tienen parte de área urbana de la ciudad denominándose "conurbados" al AUCM.

En lo que va del siglo XX, por ende, la ciudad de México ha experimentado un acelerado proceso de crecimiento que la ha transformado de una pequeña ciudad de poco más de 300 mil habitantes en una de las metrópolis más pobladas del planeta. 
Cuarta etapa: megalópolis emergente (1980 a 2010)

En las postrimerías del siglo xx los sistemas de organización territorial han experimentado una importante transformación caracterizada por la suburbanización de importantes áreas y la intensa integración de núcleos urbanos anteriormente aislados. Surgen, así, tejidos urbanos metropolitanos policéntricos que concentran estructuras y relaciones sociales más complejas constituyendo verdaderos subsistemas de ciudades altamente integrados. Se denomina megalópolis al más avanzado de este tipo de interrelaciones urbanas que surge de la unión o traslape de dos o más áreas metropolitanas.

En una investigación para delimitar las zonas metropolitanas de la región centro de México para 1980 se identifican las siguientes áreas: a) área metropolitana de la ciudad de México; b) área metropolitana de Toluca; c) área metropolitana de Puebla; y, d) área metropolitana de Cuernavaca-Cuautla (Negrete y Salazar, 1986).

El área metropolitana de la ciudad de México (AMCM) está constituida por las 16 delegaciones del Distrito Federal y 21 municipios del estado de México, como se vio anteriormente. El área metropolitana de Toluca la forman los municipios de Toluca, Lerma, Metepec, Zinacantepec, San Mateo Ateneo y Mexicalzingo. El área metropolitana de Puebla se extiende hasta Tlaxcala quedando constituida por Puebla, San Pedro Cholula y San Pablo del Monte (en Puebla) y en Tlaxcala por Amozoc, San Miguel Xoxtla, Xichohtzingo, Zacatelco, San Martín Texmelucan, siendo de transición hasta constituirse como metropolitanos Huejotzingo, Xicoténcatl, Tenancingo y Cuautlancingo. Finalmente, CuernavacaCuautla se encuentran conformando pequeñas áreas metropolitanas con Temixco, la primera, y Yautepec, la segunda. En cuanto se integre a una de ellas el municipio de Jiutepec se constituirán en una única área metropolitana.

Para nuestros propósitos lo más relevante es que las áreas metropolitanas de la ciudad de México y la de Toluca pueden incluir indistintamente al municipio de Huixquilucan. Como se considera formando parte de la primera, se podría extender el AMCM hasta abarcar Lerma y Toluca. En otras palabras, ambas están unidas o se traslapan, constituyendo técnicamente un conglomerado megalopolitano que surge a partir de 1980 y que por ser la capital la urbe principal, se puede denominar megalópolis de la ciudad de México.

Considerando los casi cinco siglos de existencia de la ciudad de México, puede parecer que su metamorfosis de una pequeña 
ciudad a una gran megalópolis es resultado de un largo proceso de carácter secular. No obstante, desde el siglo XVI al XIX experimenta únicamente un lento crecimiento aumentando su población de 30 mil habitantes en 1522 a 300 mil en 1884 . Es durante el siglo XX en que se transforma de una pequeña ciudad de 344 mil personas en 1900 en un conglomerado megalopolitano de cerca de 22 millones en 1988.

Finalmente, ha sido proyectado que hacia el año 2010 las áreas metropolitanas de Puebla y Cuernavaca se unirán al conglomerado megalopolitano que tendrá al menos 31 millones de habitantes (G. Garza, 1987: 419).

\section{Conclusiones}

En el transcurso del siglo Xx México ha experimentado un significativo desarrollo económico y una acelerada urbanización. En este proceso ocurre un cambio radical en la estructura económica nacional y el sector primario se convierte en un mero apéndice del secundario y terciario, reduciendo su participación en el producto nacional a $9.2 \%$ en 1985 . La expansión de la actividad industrial, comercial y de servicios es esencialmente urbana y ante el considerable aumento de la población total, sucede una gran multiplicación del número de ciudades y un aumento del tamaño de las existentes.

La característica fundamental del sistema de 274 ciudades en 1988 es su carácter preeminente. Se observa una elevada concentración económica y demográfica en la ciudad de México que absorbe $37 \%$ de la población urbana nacional y alrededor de $45 \%$ del PIB total del país. Aunque la sola presencia de la ciudad de México le conferiría un carácter metropolitano a la urbanización, este fenómeno se ve fortalecido por el surgimiento de otras 26 ciudades en diferentes etapas de metropolitanismo, entre las que destacan Guadalajara, Monterrey, Puebla, León y Torreón, que junto con la capital para 1980 representan $55.2 \%$ de la población urbana total.

No obstante que ha ocurrido cierta dispersión relativa de la población del país, a partir de 1980 se inicia un nuevo ámbito de concentración al emerger un conglomerado megalopolitano en torno a la ciudad de México. Hacia el año 2010 se le anexarán las áreas metropolitanas de Puebla y Cuernavaca para consolidar un complejo mosaico megalopolitano que tendrá al menos 31 millones de habitantes.

A partir de 1982 el crecimiento económico se ha interrumpí- 
do, pero no así el proceso de urbanización. Éste sigue una dinámica propia y a reserva de confirmarlo con los datos del censo de 1990 , todo parece indicar que las ciudades siguen creciendo. Las únicas ciudades que crecen económicamente son algunas de la frontera con Estados Unidos de América gracias al auge de la industria maquiladora, tales como Tijuana, ciudad Juárez y Matamoros, y otras turísticas como Cancún y Puerto Vallaría.

El escenario socioeconómico del sistema de ciudades mexicanas depende fundamentalmente de las posibilidades reales de vencer la crisis y retomar las anteriores tasas de crecimiento económico. De no hacerlo en un futuro previsible, y todo parece indicar que así será, la situación económica, social y urbanística de la población que habita en las urbes mexicanas podría ser muy sombría.

\section{Bibliografía}

Armostrong, W. y McGee, T.G. (1985), Theatres of Accumulation. Studies in Asian and Latin America Urbanization, Methuen, Londres y Nueva York.

Carmona de la Peña (1979), "Reflexiones sobre el desarrollo económico de México, 1929-1979-2. En Gaceta UNAM, vol, III, suplemento núm. 3, México.

Garza, Gustavo (1985), El proceso de industrialización de la Ciudad de México, 1821-1970, El Colegio de México. et al. (comps. 1987), Atlas de la Ciudad de México, El Colegio de México, Departamento del Distrito Federal, México.

(1987), "El futuro de la ciudad de México: megalópolis emergente", en G. Garza et al., op. cit.

y Aguilar, E. (1988), "Evolución industrial de la ciudad de México", en Concamin, Historia y Desarrollo Industrial de México, MéxiCo, D.F.

Harris, Nigel (1988), "Urbanization: An Economic Overview of Some of the Issues", en Habitat International, vol. 12, núm. 3, Gran Bretaña.

INEGI (1985), Sistema de cuentas nacional de México, Estructura económica regional, Producto Interno Bruto por entidad federativa, 19701975-1980, Instituto Nacional de Estadística, Geografía e Informática, México, D.F.

(1988), Producto Interno Bruto por entidad federativa, 1980-1985 (no publicado), México, D.F.

Negrete, Ma. Eugenia y Héctor Salazar (1987), "Dinámica de crecimiento de la población de la ciudad de México: 1900-1980", en G. Garza et al., Atlas de la Ciudad de México, El Colegio de México, DDF, México.

(1986), “Zonas Metropolitanas en México, 1980", Estudios Demo- 
gráficos y Urbanos, vol. 1, núm. 1, enero-abril, El Colegio de México. Unikel, L., Ruiz, Ch., C. y Garza G. (1976), El desarrollo urbano de México, El Colegio de México.

Unikel, L. (1977), "Urbanization in Mexico: Process, Implications, Policies and Prospects", en, Sidney Goldstein y David F. Sly (Eds.), Patterns of Urbanization: comparative Country Studies, Ordina Editores, Bélgica (vol. 2).

ONU (1987), The Prospects of World Urbanization, Nueva York. 
